\title{
Comparative Genomic Analysis of Delftia tsuruhatensis MTQ3 and the Identification of Functional NRPS Genes for Siderophore Production
}

\author{
Haimeng Guo, ${ }^{1}$ Yanan Yang, ${ }^{1}$ Kai Liu, ${ }^{1}$ Wenfeng $\mathrm{Xu}^{2}{ }^{2}$ Jianyong Gao, ${ }^{3}$ \\ Hairong Duan, ${ }^{3}$ Binghai Du, ${ }^{1}$ Yanqin Ding, ${ }^{1}$ and Chengqiang Wang ${ }^{1}$ \\ ${ }^{1}$ College of Life Sciences/Shandong Key Laboratory of Agricultural Microbiology, Shandong Agricultural University, Taian, China \\ ${ }^{2}$ State Key Laboratory of Nutrition Resources Integrated Utilization, Linyi, China \\ ${ }^{3}$ GENEWIZ, Suzhou, China
}

Correspondence should be addressed to Yanqin Ding; dyq@sdau.edu.cn and Chengqiang Wang; wangcq@sdau.edu.cn

Received 28 April 2016; Revised 17 September 2016; Accepted 3 October 2016

Academic Editor: Dilfuza Egamberdieva

Copyright (C) 2016 Haimeng Guo et al. This is an open access article distributed under the Creative Commons Attribution License, which permits unrestricted use, distribution, and reproduction in any medium, provided the original work is properly cited.

Plant growth-promoting rhizobacteria (PGPR) are a group of rhizosphere bacteria that promote plant growth. Delftia tsuruhatensis MTQ3 is a member of PGPR that produces siderophores. The draft genome sequence of MTQ3 has been reported. Here, we analyzed the genome sequence of MTQ3 and performed a comparative genome analysis of four sequenced Delftia strains, revealing genetic relationships among these strains. In addition, genes responsible for bacteriocin and nonribosomal peptide synthesis were detected in the genomes of each strain. To reveal the functions of NRPS genes in siderophore production in D. tsuruhatensis MTQ3, three NRPS genes were knocked out to obtain the three mutants MTQ3- 1941 , MTQ3- $\Delta 1945$, and MTQ3- $\Delta 1946$, which were compared with the wild-type strain. In qualitative and quantitative analyses using CAS assay, the mutants failed to produce siderophores. Accordingly, the NRPS genes in MTQ3 were functionally related to siderophore production. These results clarify one mechanism by which plant growth is promoted in MTQ3 and have important applications in agricultural production.

\section{Introduction}

Plant growth-promoting rhizobacteria (PGPR) could promote plant growth by a variety of mechanisms, such as siderophore production [1], antibiotics secretion, phytohormone generation, and the induction of systemic resistance $[2,3]$. PGPR have an important value in agricultural production.

Iron is essential to the growth of plants, but soluble iron is often insufficient in soil. Siderophores, which are low-molecular-weight molecules with a high affinity for ferric iron [4] secreted by PGPR, can mitigate that limitation, to some extent. Many microorganisms, including bacteria and fungi, can secrete siderophores [5]. In general, siderophores can be separated into two types based on their biosynthetic mechanism. One type depends on the nonribosomal peptide synthetase (NRPS) pathway, and the other is NRPS-independent [6].
Delftia has the ability to biodegrade organic pollutants, such as aniline [7], phenolic compounds [8], 2,4-dichlorophenoxyacetic acid (2,4-D) [9], and acetochlor. Delftia tsuruhatensis MTQ3 (MTQ3 for short) has been isolated by our group from the rhizosphere of tobacco in Guizhou, China. As an environmentally friendly PGPR, MTQ3 exhibits the potential to produce siderophores. Although species in the genus Delftia have been described as PGPR [10], their ability to produce siderophores has not been verified. The draft genome sequence of MTQ3 was formerly reported [11], but the mechanism of siderophore production is unclear.

In this work, a comparative genomic analysis of MTQ3 and the related genome sequences of Delftia sp. Cs1-4, D. acidovorans SPH-1, and D. tsuruhatensis 391 was performed. We characterized the genetic differences between the four Delftia strains. According to the genome annotation of MTQ3, a gene cluster that included three NRPS genes on scaffold 2 
TABLE 1: Bacterial strains and plasmids used in this work.

\begin{tabular}{|c|c|c|}
\hline Strain/plasmid & Relevant characteristics & Source \\
\hline \multicolumn{3}{|l|}{ D. tsuruhatensis } \\
\hline $\mathrm{MTQ}_{3}$ & Wild-type strain, Rif $^{\mathrm{r}}, \mathrm{Km}^{\mathrm{s}}$ & [11] \\
\hline $\mathrm{MTQ}_{3}-\Delta 1941$ & D. tsuruhatensis $\mathrm{MTQ}_{3-} \Delta 1941, \mathrm{Rif}^{\mathrm{r}}, \mathrm{Km}^{\mathrm{r}}$ & This work \\
\hline $\mathrm{MTQ}_{3}-\Delta 1945$ & D. tsuruhatensis $\mathrm{MTQ}_{3-} \Delta 1945, \mathrm{Rif}^{\mathrm{r}}, \mathrm{Km}^{\mathrm{r}}$ & This work \\
\hline $\mathrm{MTQ}_{3}-\Delta 1946$ & D. tsuruhatensis $\mathrm{MTQ}_{3-} \Delta 1946, \mathrm{Rif}^{\mathrm{r}}, \mathrm{Km}^{\mathrm{r}}$ & This work \\
\hline \multicolumn{3}{|c|}{ 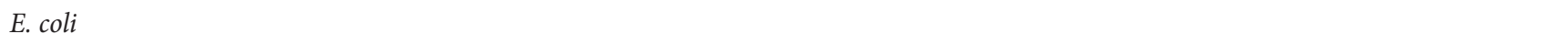 } \\
\hline $\mathrm{DH} 5 \alpha$ & Host of recombinant plasmids & TransGen \\
\hline \multicolumn{3}{|l|}{ Plasmids } \\
\hline pJQ200SK & Suicide plasmid carrying $s a c B, \mathrm{Gm}^{\mathrm{r}}$, selecting double crossover $\mathrm{MTQ}_{3}$ & {$[12]$} \\
\hline pk-1941 & pJQ200SK carrying Km and the fragment of orf-1941, $\mathrm{Gm}^{\mathrm{r}}, \mathrm{Km}^{\mathrm{r}}$ & This work \\
\hline pk-1945 & pJQ200SK carrying Km and the fragment of orf-1945, $\mathrm{Gm}^{\mathrm{r}}, \mathrm{Km}^{\mathrm{r}}$ & This work \\
\hline pk-1946 & pJQ200SK carrying $\mathrm{Km}$ and the fragment of orf-1946, $\mathrm{Gm}^{\mathrm{r}}, \mathrm{Km}^{\mathrm{r}}$ & This work \\
\hline pUC4K & Carrying Km cassette (Pst $\mathrm{I}), \mathrm{Km}^{\mathrm{r}}$ & {$[13]$} \\
\hline pRK2013 & Helper plasmid used in triparental mating, $\mathrm{Km}^{\mathrm{r}}$, Rif $^{\mathrm{s}}$ & {$[14]$} \\
\hline pGEM-T easy & TA cloning vector, $\mathrm{Amp}^{\mathrm{r}}$ & Promega \\
\hline
\end{tabular}

TABLE 2: Oligonucleotides used in this study.

\begin{tabular}{lrc}
\hline Primers & Sequence $\left(5^{\prime}-3^{\prime}\right)$ & Purpose \\
\hline J1941F & GGACTAGTCTTTGGCGTGCCGATGT $($ Spe I $)$ & Cloning the fragment of orf-1941 \\
J1941R & CCGCTCGAGTCGTTGGCGATGAGGTTGC $($ Xho I $)$ & Cloning the fragment of orf-1945 \\
J1945F & GGACTAGTCCCTGAACGATCTCGATTCCC $($ Spe I $)$ & Cloning the fragment of orf-1946 \\
J1945R & CCGCTCGAGCATAGGTGCCACCGGCCTTG $($ Xho I $)$ & Cloning the fragment of Kna \\
J1946F & GGACTAGTGCTTCCGCTGATCGACCTCA $($ Spe I $)$ & Cloning the fragment of 16 srDNA \\
J1946R & CCGCTCGAGCGCCTTCTTCATCCTGCTCC $($ Xho I $)$ & CCCATCATCCAGCCAGAAAGTG \\
KF1 & ATAATGTCGGGCAATCAGGTGC & AGA GTT TGA TCC TGG CTC AG \\
KR1 & TAC GGC TAC CTT GTT ACG ACTT & \\
1492R &
\end{tabular}

was found. The NRPS modular organizations were predicted using the PKS/NRPS Analysis website [15]. We constructed NRPS gene knockout mutants to analyze gene function with respect to siderophore biosynthesis.

\section{Materials and Methods}

2.1. Bacterial Strains and Plasmids. Bacterial strains and plasmids used in this study are presented in Table 1. D. tsuruhatensis MTQ3 was used as the wild-type strain to conduct mutants. Three NRPSs (AA671_12415, AA671_12425, and AA671_12430) were knocked out to generate three mutants:

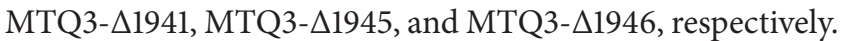
Escherichia coli strains were used for the construction of plasmids.

2.2. DNA Extraction. Chromosomal DNA of MTQ3 was extracted from $1 \mathrm{~mL}$ of overnight culture using the TIANamp Bacteria DNA Kit (TIANGEN, Beijing, China) according to the manufacturer's instructions.
2.3. Primers and Gene Amplification. All primers used in this study are presented in Table 2 . The target genes orf1941 (AA671_12415), orf-1945 (AA671_12425), and orf-1946 (AA671_12430) were amplified from the genomic DNA of MTQ3 by PCR using relevant primers. PCR fragments were electrophoresed, excised, and purified using the TIANgel Midi Purification Kit (TIANGEN) [16].

2.4. Molecular Phylogenetic Analysis. To make phylogenetic inferences, the 16S rRNA gene was amplified from the genomic DNA using the universal bacterial 16S rRNA gene primers $27 \mathrm{~F}$ and $1492 \mathrm{R}$. PCR products were sequenced by GENEWIZ (Jiangsu, China). The 16S rRNA gene sequence was Blast-searched $[17,18]$ against the NCBI database [19] to identify homologous sequences from other species. A phylogenetic tree were generated using MEGA5 [20].

2.5. Comparative Genome Analysis. The three Delftia genomes Delftia sp. Cs1-4 (NC-015563), D. acidovorans SPH-1 (NC-010002), and D. tsuruhatensis 391 (JNWH00000000) 


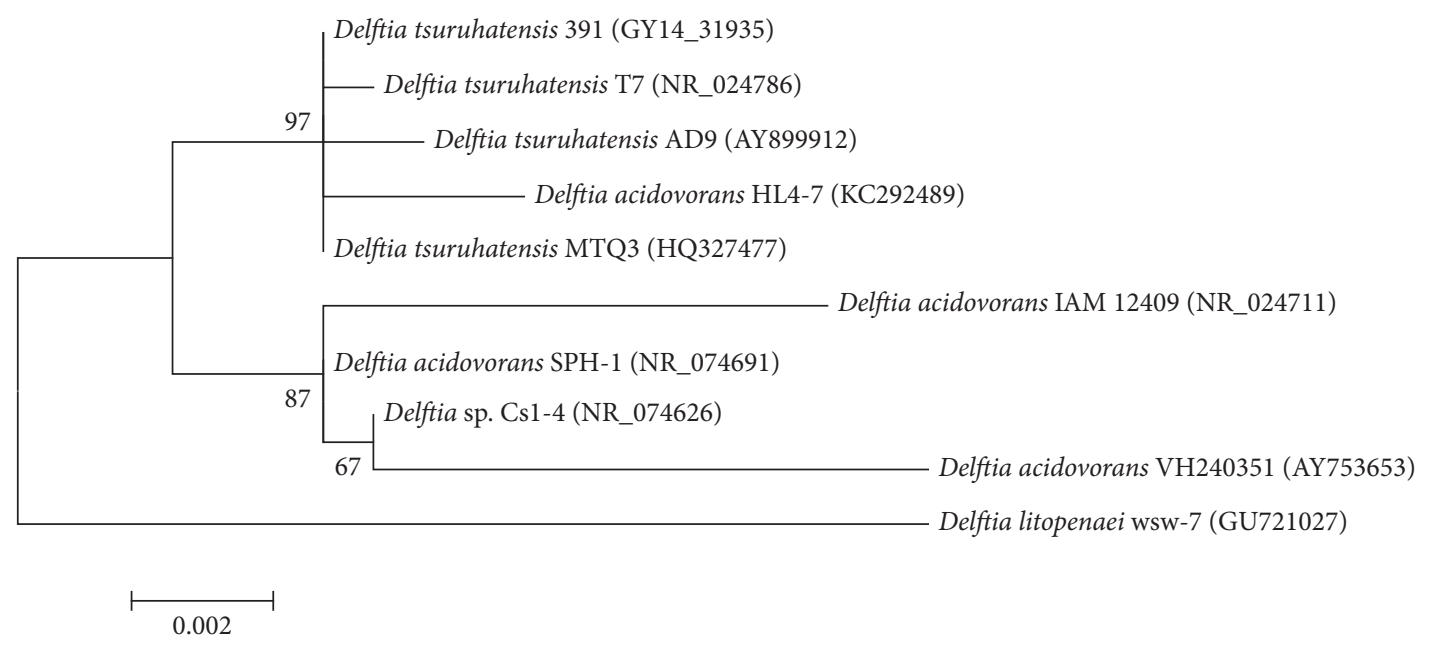

FIGURE 1: Phylogenetic tree based on the 16S rRNA gene sequence of MTQ3 and related strains. The phylogenetic tree was constructed using the maximum likelihood method with 1000 bootstrap replications. GenBank accession numbers are presented in brackets next to the species names.

obtained from GenBank were used for a comparative genome analysis with MTQ3 (LCZH00000000). The clusters of orthologous groups (COG) functional categories were analyzed by exploring all predicted proteins in the COG database using BLASTP [21]. Nonribosomal peptide and polyketide synthesis gene clusters were recognized using anti-SMASH (http://antismash.secondarymetabolites.org/help.html) [22], and their structures were compared to those of other known clusters.

2.6. Gene Knockout. All molecular genetic procedures for the genes orf-1941, orf-1945, and orf-1946 were performed according to the methods described in [23].

2.7. Medium and Cultivation. For plasmid construction, E. coli strains were cultured in Luria-Bertani (LB) medium with gentamycin $(50 \mu \mathrm{g} / \mathrm{mL})$ or kanamycin $(100 \mu \mathrm{g} / \mathrm{mL})$, as needed, at $37^{\circ} \mathrm{C}$. D. tsuruhatensis MTQ3 and the mutants were grown in LB medium with rifampicin $(10 \mu \mathrm{g} / \mathrm{mL})$. The $5 \%$ sucrose plus LB plates with kanamycin and rifampicin were used to screen the recombinant strains. A CAS-agar plate [24] was used to qualitatively detect siderophores. For the quantitative analysis of siderophores, sucrose-asparagine (SA) medium was necessary, which included (per liter) $20 \mathrm{~g}$ of sucrose, $2.0 \mathrm{~g}$ of L-asparagine, $0.5 \mathrm{~g}$ of $\mathrm{K}_{2} \mathrm{HPO}_{4}$, and $0.5 \mathrm{~g}$ of $\mathrm{MgSO}_{4} \cdot 7 \mathrm{H}_{2} \mathrm{O}$ [25].

2.8. Qualitative and Quantitative Analyses of Siderophores. Single clones of strains MTQ3, MTQ3- $\Delta 1941$, MTQ3- 1945 , and MTQ3- $\Delta 1946$ were cultivated in LB plates at $37^{\circ} \mathrm{C}$ overnight. Then, the bacterial lawn was inoculated on a CAS-agar plate for cultivation at $37^{\circ} \mathrm{C}$ for 2-3 days, and the development of a color ring was monitored.

Single clones of MTQ3, MTQ3- 1 1941, MTQ3- 1945 , and MTQ3- $\Delta 1946$ were inoculated into $10 \mathrm{~mL}$ of sucroseasparagine (SA) liquid medium and incubated at $180 \mathrm{rpm}$ and $37^{\circ} \mathrm{C}$ for two days. SA liquid medium [25] contains
$20 \mathrm{gL}^{-1}$ sucrose, $2.0 \mathrm{~g} \mathrm{~L}^{-1}$ L-asparagine, $0.5 \mathrm{~g} \mathrm{~L}^{-1} \mathrm{~K}_{2} \mathrm{HPO}_{4}$, and $0.5 \mathrm{~g} \mathrm{~L}^{-1} \mathrm{MgSO}_{4} \cdot 7 \mathrm{H}_{2} \mathrm{O}$. The supernatants of liquid cultures $\left(A_{s}\right)$ were collected by centrifugation at $10000 \mathrm{rpm}$ for $15 \mathrm{~min}$ and then mixed with the CAS assay solution (at a volume ratio of $1: 1$ ) [26]. Uninoculated SA liquid medium was also treated following the same protocol as a reference $\left(A_{r}\right)$. After a 1-hour reaction period, the absorbances of $A_{s}$ and $A_{r}$ were detected at $630 \mathrm{~nm}$ [27]. Siderophore units were estimated as $\left[\left(A_{r}-A_{s}\right) / A_{r}\right] \times 100=$ siderophore units $(\%)$ [24]. Units not exceeding 10 were regarded as negative and no color change was found in the mixture.

\section{Results}

3.1. Phylogenetic Analyses. A phylogenetic tree was constructed based on the 16S rRNA sequences of Delftia spp. [28] in MEGA5 (Figure 1). These results suggested that strain MTQ3 had high homology with D. tsuruhatensis.

3.2. Comparative Analysis with Strains Cs1-4, SPH-1, and 391. The general features of the four genomes are summarized in Table 3 . There was clear variation in genome size. Specifically, the genome size varied from $5.7 \mathrm{Mb}$ in MTQ3 to $6.7 \mathrm{Mb}$ in SPH-1. Chromosomal coding DNA sequences (CDS) varied between 4103 in strain 391 and 6040 in SPH-1. The $(G+C)$ mol\% of the species shared a mean value of $66.60 \%$ and no strain differed from the mean value by $>0.3 \%$. These results indicated a genetic relationship between the species, to some extent.

To compare these genomes, the orthologous and unique genes among the four genomes were analyzed (Figure 2) [21]. The orthologous genes are contained in all strains, while the unique genes are owned by only one strain. A total of 2540 orthologous genes were shared and represented $51.05 \%$, $43.34 \%, 42.05 \%$, and $61.91 \%$ of all genes in MTQ3, Cs1-4, SPH-1, and 391, respectively. MTQ3 shared 4470, 4414, and 2782 orthologous genes with Cs1-4, SPH-1, and strain 391, 
TABLE 3: General features of D. tsuruhatensis MTQ3 and other related genomes.

\begin{tabular}{lcccc}
\hline & D. tsuruhatensis & Delftia sp. & D. acidovorans & D. tsuruhatensis \\
& MTQ3 & Cs1-4 & SPH-1 & $6,732,149$ \\
\hline Genome size & $5,737,182$ & $6,685,842$ & $6,767,514$ & 4103 \\
CDS number & 4976 & 5861 & 6040 & $66.30 \%$ \\
G + C percentage & $66.90 \%$ & $66.71 \%$ & $66.47 \%$ & 76 \\
RNA number & 92 & 98 & 98 & \\
\hline
\end{tabular}

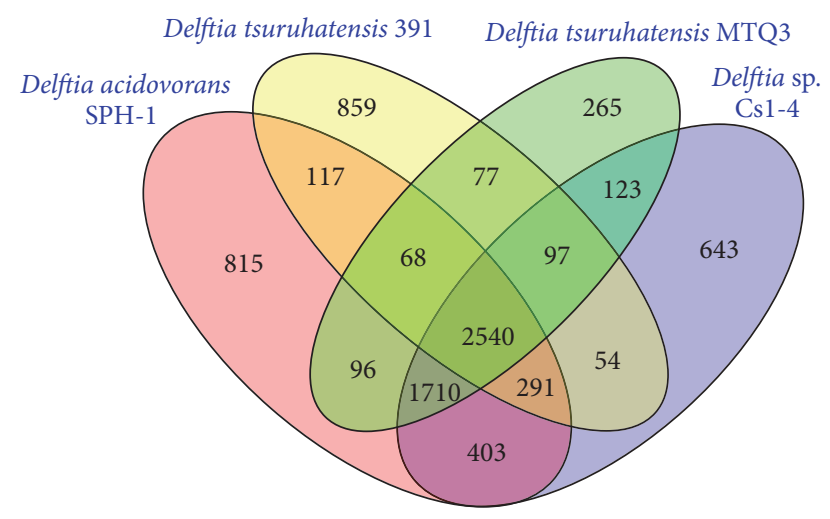

FIGURE 2: Comparison of the gene contents of MTQ3, Cs1-4, SPH-1, and strain 391.

respectively. Meanwhile, MTQ3 possessed the least unique genes and accounted for $5.14 \%$ of all genes in its genome, but strain 391 contained the most unique genes and accounted for $20.94 \%$ in its genome. That might indicate that the genes of MTQ3 presented more conserved core genome for $D$. tsuruhatensis.

Based on the COG-based analysis, the genes of these four genomes showed some similarities with respect to the distributions of COG categories (Figure 3). For the four genomes, genes related to transcription, amino acid transport and metabolism, and lipid transport and metabolism (COG categories $\mathrm{K}, \mathrm{E}$, and I, resp.) were relatively abundant functional categories, in addition to $\mathrm{R}$ and $\mathrm{S}$, which represent general predicted functions and unknown functions, respectively, and provide little information regarding protein function [29]. The genome of MTQ3 included a larger proportion of genes involved in carbohydrate transport and metabolism compared with the other three genomes.

Bacteriocins, which are antimicrobial peptides or proteins produced by bacteria, could enhance environmental adaption. Enzymes related to the synthesis of nonribosomal peptides (NRP) and polyketides (PK) are modular and composed of a series of domains including adenylation, thiolation, condensation, and esterification domains [29]. We compared the NRPS gene cluster between the four genomes of Delftia, which are summarized in Figure 4. Bacteriocin synthetic gene clusters are also listed in Figure 4(b). The query sequence refers to the sequence of MTQ3. These results showed that not all Delftia genomes have identical clusters of NRPS and bacteriocin synthesis genes. These differences

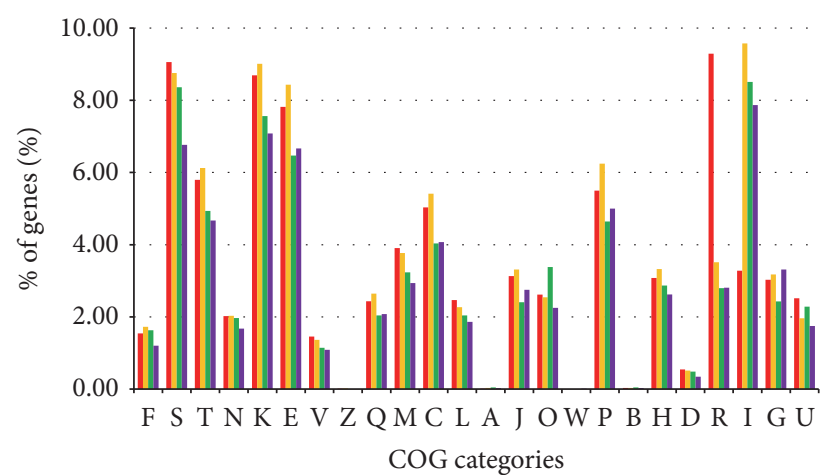

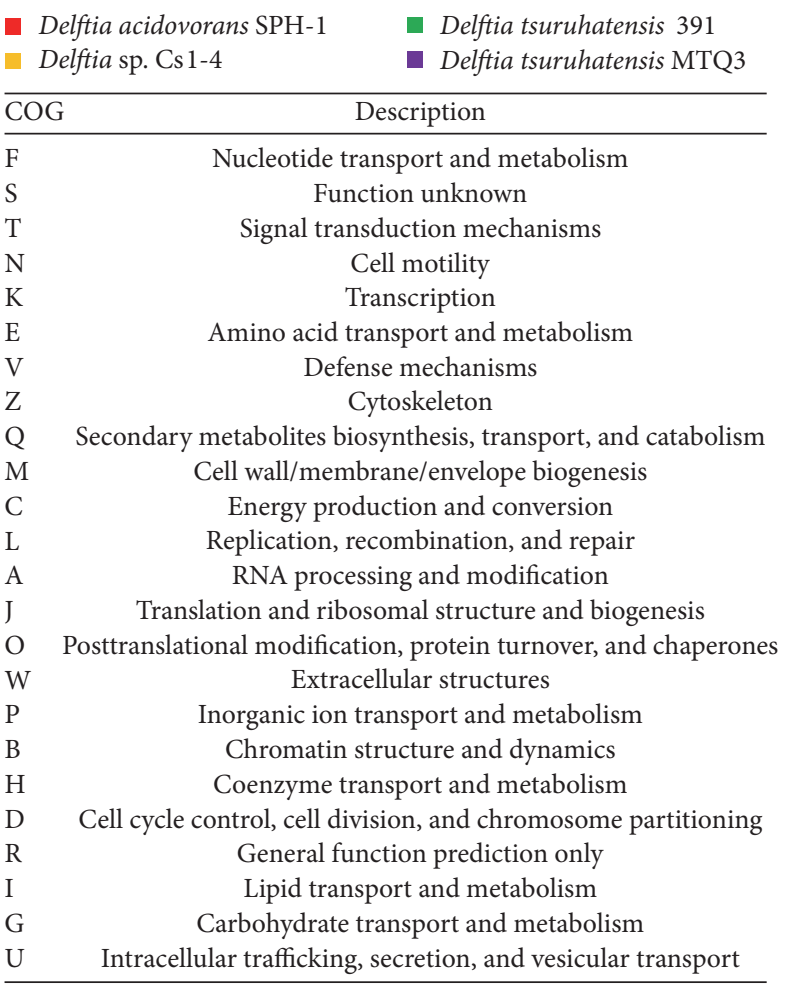

FIGURE 3: COG functional categorization of sequenced Delftia genomes.

may reflect adaptations of the strains to their specific environments.

3.3. The Knockout of NRPSs in MTQ3. To reveal the function of the NRPS genes (Figure 4(a)), gene knockouts were 


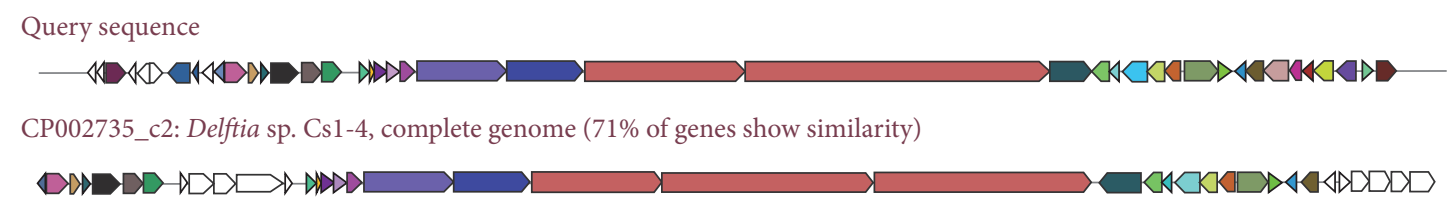

CP000884_c3: Delftia acidovorans SPH-1, complete genome (65\% of genes show similarity)

(a)

Query sequence

AGYX01000005_c1: Delftia acidovorans CCUG 274B acHMQ-supercont1.4.C5, whole... (24\% of genes show similarity)

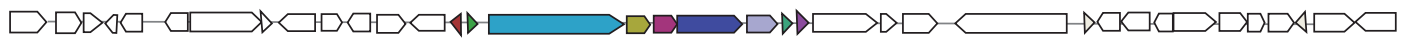

CP000884_c2: Delftia acidovorans SPH-1, complete genome (24\% genes show similarity)

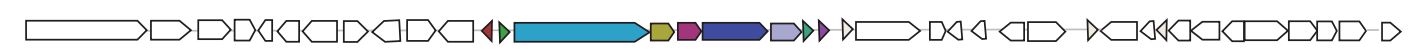

JOUB01000003_c1: Delftia acidovorans strain 2167 DR66. Contig240, whole geno ... (24\% of genes show similarity)

$\square \bowtie\langle\triangleright \square \bowtie \triangleright$

CP002735_c3: Delftia sp.Cs1-4, complete genome (24\% of genes show similarity)

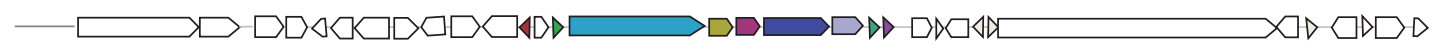

AGYY01000003_c1: Delftia acidovorans CCUG 15835 acHMM-supercont1.2.C3, whol... (21\% of genes show similarity)

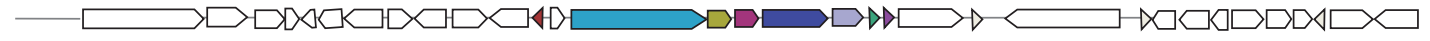

(b)

FIGURE 4: Nonribosomal peptide and polyketide synthesis clusters. (a) NRPS gene cluster. (b) Bacitracin synthesis cluster. The query sequence refers to the sequence of MTQ3 (http://antismash.secondarymetabolites.org/help.html).

performed. The amplified target fragments of three NRPSs were ligated into the pGEM-T easy vector between sites Spe I and Xho I, which were then religated to the suicide plasmid pJQ200SK using the same restriction endonucleases for cutting. The resulting suicide plasmids were then linearized by Pst I. Km fragments were cloned from the plasmid pUC4k by Pst I digestion and then ligated into the above linearized suicide plasmids. Finally, the resulting three plasmids pk-1941, pk-1945, and pk-1946 were transformed into E. coli DH5 $\alpha$ [30]. With the help of plasmid pRK2013, triparental mating [31] was used to generate the recombinant strains MTQ3$\Delta 1941$, MTQ3- $\Delta 1945$, and MTQ3- $\Delta 1946$ (Figure 5). The three mutants were selected by $5 \%$ sucrose plus LB plates with kanamycin and rifampicin and further confirmed by PCR using primers for $\mathrm{Km}$ and sequencing.

3.4. Qualitative and Quantitative Analyses of Siderophores. On the CAS-agar plates, we observed an orange halo around the colony of MTQ3 after 2-3 days of incubation, but no orange ring around the mutants (Figure 6). The presence of the orange ring suggested that MTQ3 can produce siderophores to chelate iron in the medium, thus resulting in a color change of the medium surrounding the colony. The mutants lost the ability to produce siderophores.

The quantitative measurements of siderophores (Table 4) indicated that the siderophore units of the wild-type strain
TABLE 4: Quantification of siderophores.

\begin{tabular}{lc}
\hline Strain & Siderophore units (\%) \\
\hline MTQ3 & $47.8 \pm 0.87$ \\
MTQ3- $\Delta 1941$ & $1.69 \pm 0.64$ \\
MTQ3- $\Delta 1945$ & $0.72 \pm 0.48$ \\
MTQ3- $\Delta 1946$ & $3.86 \pm 0.48$ \\
\hline
\end{tabular}

MTQ3 were 47.8\%. However, production levels by MTQ3$\Delta 1941$, MTQ3- $\Delta 1945$, and MTQ3- $\Delta 1946$ were just less than $5 \%$, which were regarded as negative results. In addition, we observed a color change from blue to orange when mixing the MTQ3 culture with the CAS assay solution, but this phenomenon was not observed for MTQ3- $\Delta 1941$, MTQ3- $\Delta 1945$, and MTQ3- 1946 . This further demonstrated that NRPS

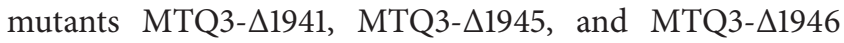
failed to produce siderophores.

\section{Discussion}

In this study, D. tsuruhatensis MTQ3 was examined as a PGPR. The phylogenetic analyses showed that MTQ3 has high homology with D. tsuruhatensis. General features and a comparative genomic analysis with Delftia Cs1-4, SPH-1, and strain 391 suggested that D. tsuruhatensis MTQ3 shows 


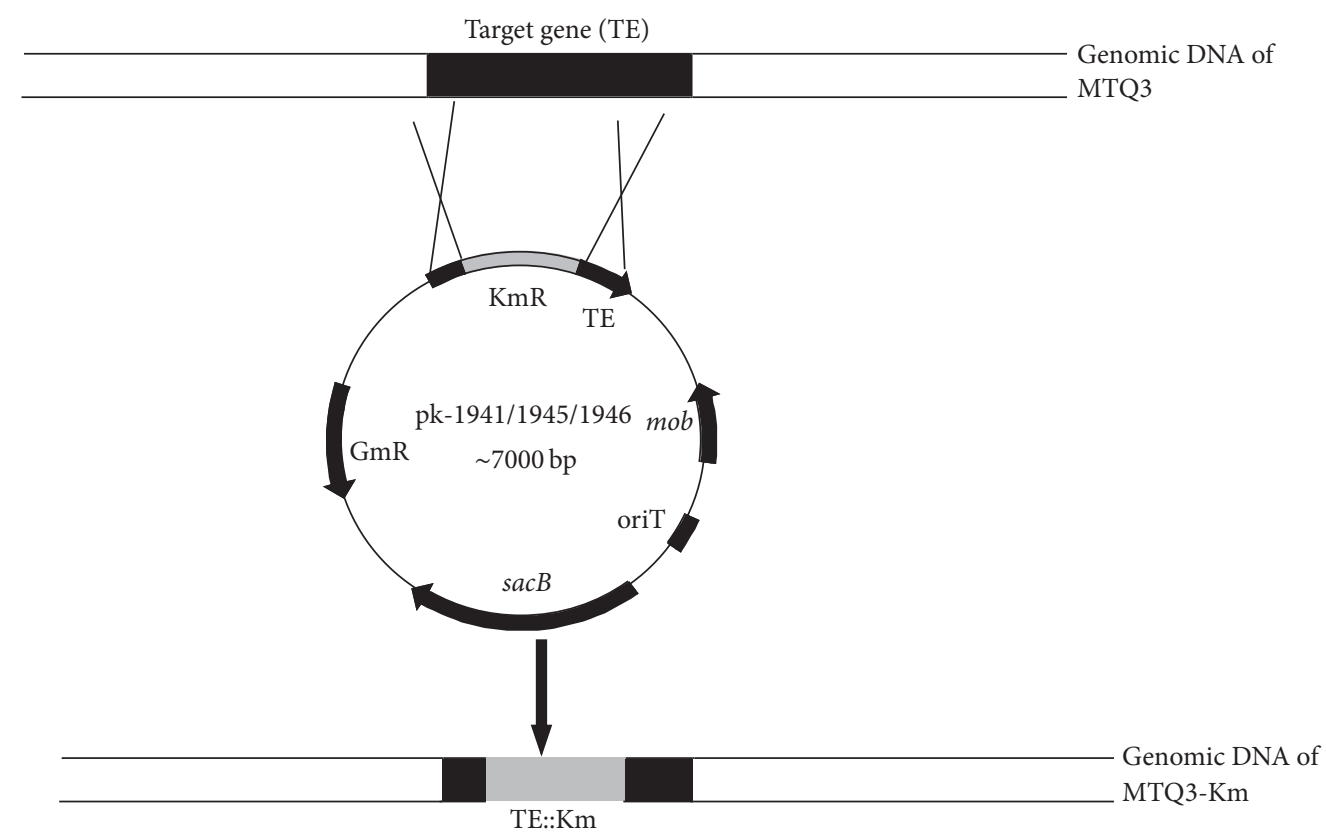

FIGURE 5: Knockout target genes with the Km cassette. The target genes orf-1941, orf-1945, and orf-1946 were amplified from the genomic DNA of MTQ3, after a series of enzyme digestions and ligation to form the recombinant plasmids pK-1941, pK-1945, and pK-1946. Triparental mating was used to generate homologous recombinant strains.

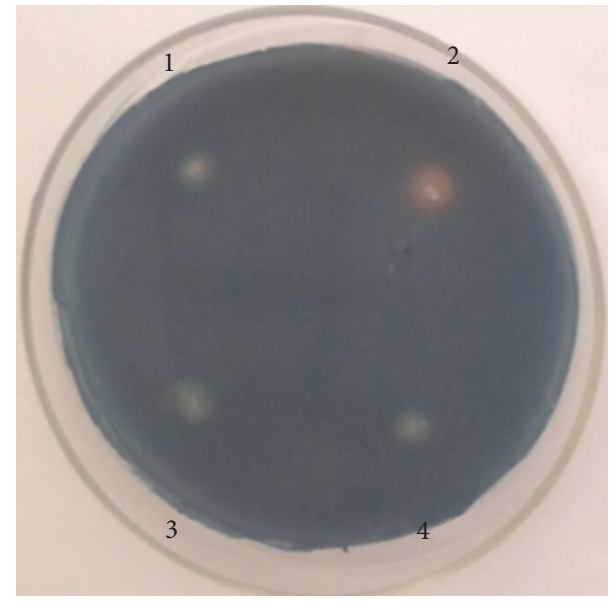

FIGURE 6: Qualitative analysis of siderophores on the CAS-agar plate. The bacterial lawn was inoculated on the CAS-agar plate for cultivation at $37^{\circ} \mathrm{C}$ for 2-3 days, followed by monitoring for a color ring. (1) $\mathrm{MTQ}_{3}-\Delta 1941$, (2) MTQ3, (3) $\mathrm{MTQ}_{3}-\Delta 1945$, and (4) $\mathrm{MTQ}_{3}$ $\Delta 1946$.

some similarities with respect to COG categories, but the proportions are somehow different. MTQ3 contains a larger proportion of genes involved in carbohydrate transport and metabolism, which indicates its better potential for carbohydrate utilization. For secondary metabolite prediction using anti-SMASH, the gene clusters of NRPS and bacteriocin synthesis genes are not identical in the Delftia genomes. The different features of the four genomes may be explained by adaptations of strains to their specific environments.
As PGPR, MTQ3 exhibits the potential to produce siderophores. To determine the genes involved in siderophore production, three NRPS genes orf-1941, orf-1945, and orf1946 of MTQ3 were figured out to test the function. They belong to one gene cluster, the genes of which show $85 \%$ similarity to the nonribosomal peptide metallophore, delftibactin [32, 33]. Compared with the wild-type strain MTQ3, three obtained mutants lost their ability to produce siderophores. These results indicated that siderophores could be biosynthesized by the NRPS modular multienzymes in MTQ3. Siderophores could improve the absorption of iron by plants, therefore promoting growth [4]. The ability of siderophores produced by bacteria to repress phytopathogens could be of significant importance in agriculture. This may explain why D. tsuruhatensis MTQ3 could stimulate plant growth. To the best of our knowledge, this is the first study to verify the gene cluster for siderophore production in $D$. tsuruhatensis. Meanwhile, the PGPR of MTQ3 may have important applications in agriculture.

\section{Competing Interests}

All authors declare that they have no competing interests.

\section{Authors' Contributions}

Haimeng Guo and Yanan Yang were equal contributors to this work.

\section{Acknowledgments}

This work was supported by the National Science and Technology Pillar Program of China (2014BAD16B02), the Science 
and Technology Major Projects of Shandong Province (2015ZDXX0502B02), and the National Natural Science Foundation of China (31100005).

\section{References}

[1] A. Beneduzi, A. Ambrosini, and L. M. P. Passaglia, "Plant growth-promoting rhizobacteria (PGPR): their potential as antagonists and biocontrol agents," Genetics and Molecular Biology, vol. 35, no. 4, pp. 1044-1051, 2012.

[2] G. H. Zheng, J. C. Wang, and X. H. Wang, "Mechanisms of plant growth promoting rhizobacteria," JIANGXI Science, vol. 30, no. 4, pp. 454-458, 2012.

[3] M. Ahemad and M. Kibret, "Mechanisms and applications of plant growth promoting rhizobacteria: current perspective," Journal of King Saud University-Science, vol. 26, no. 1, pp. 120, 2014.

[4] M. Miethke and M. A. Marahiel, "Siderophore-based iron acquisition and pathogen control," Microbiology and Molecular Biology Reviews, vol. 71, no. 3, pp. 413-451, 2007.

[5] Y. Zhang, W. Zhang, X. Chen, and F. Zhang, "The structure, function and progress of siderophores produced by bacteria," Chinese Journal of Health Laboratory Technology, vol. 22, no. 9, pp. 2249-2251, 2012.

[6] S. M. Barry and G. L. Challis, "Recent advances in siderophore biosynthesis," Current Opinion in Chemical Biology, vol. 13, no. 2, pp. 205-215, 2009.

[7] H. Yan, X. Yang, J. Chen, C. Yin, C. Xiao, and H. Chen, "Synergistic removal of aniline by carbon nanotubes and the enzymes of Delftia sp. XYJ6," Journal of Environmental Sciences, vol. 23, no. 7, pp. 1165-1170, 2011.

[8] B. J. Jimenez, P. Reboleiro Rivas, J. G. Lopez, C. Pesciaroli, P. Barghini, and M. Fenice, "Immobilization of Delftia tsuruhatensis in macro-porous cellulose and biodegradation of phenolic compounds in repeated batch process," Journal of Biotechnology, vol. 157, no. 1, pp. 148-153, 2012.

[9] H. Yoon, S. Leibeling, C. Zhang, R. H. Müller, C. J. Werth, and J. L. Zilles, "Adaptation of Delftia acidovorans for degradation of 2,4-dichlorophenoxyacetate in a microfluidic porous medium," Biodegradation, vol. 25, no. 4, pp. 595-604, 2014.

[10] J. Han, L. Sun, X. Dong et al., "Characterization of a novel plant growth-promoting bacteria strain Delftia tsuruhatensis HR4 both as a diazotroph and a potential biocontrol agent against various plant pathogens," Systematic and Applied Microbiology, vol. 28, no. 1, pp. 66-76, 2005.

[11] Q. Hou, C. Wang, H. Guo et al., "Draft genome sequence of Delftia tsuruhatensis MTQ3, a strain of plant growth-promoting rhizobacterium with antimicrobial activity," Genome Announcement, vol. 3, no. 4, p. e00822-15, 2015.

[12] J. Quandt and M. F. Hynes, "Versatile suicide vectors which allow direct selection for gene replacement in Gram-negative bacteria," Gene, vol. 127, no. 1, pp. 15-21, 1993.

[13] J. Vieira and J. Messing, "The pUC plasmids, an M13mp7derived system for insertion mutagenesis and sequencing with synthetic universal primers," Gene, vol. 19, no. 3, pp. 259-268, 1982.

[14] D. H. Figurski and D. R. Helinski, "Replication of an origincontaining derivative of plasmid RK2 dependent on a plasmid function provided in trans," Proceedings of the National Academy of Sciences of the United States of America, vol. 76, no. 4, pp. 1648-1652, 1979.
[15] B. O. Bachmann and J. Ravel, "Chapter 8 methods for in silico prediction of microbial polyketide and nonribosomal peptide biosynthetic pathways from DNA sequence data," Methods in Enzymology, vol. 458, pp. 181-217, 2009.

[16] P. Shen, H. Chao, C. Jiang, Z. Long, C. Wang, and B. Wu, "Enhancing production of l-serine by increasing the glyA gene expression in Methylobacterium sp. MB200," Applied Biochemistry and Biotechnology, vol. 160, no. 3, pp. 740-750, 2010.

[17] S. F. Altschul, W. Gish, W. Miller, E. W. Myers, and D. J. Lipman, "Basic local alignment search tool," Journal of Molecular Biology, vol. 215, no. 3, pp. 403-410, 1990.

[18] C. Camacho, G. Coulouris, V. Avagyan et al., "BLAST+: architecture and applications," BMC Bioinformatics, vol. 10, article 421, 2009.

[19] D. A. Benson, M. Cavanaugh, K. Clark et al., "GenBank," Nucleic Acids Research, vol. 41, no. 1, pp. D36-D42, 2013.

[20] K. Tamura, D. Peterson, N. Peterson, G. Stecher, M. Nei, and S. Kumar, "MEGA5: molecular evolutionary genetics analysis using maximum likelihood, evolutionary distance, and maximum parsimony methods," Molecular Biology and Evolution, vol. 28, no. 10, pp. 2731-2739, 2011.

[21] H. Kang, X. Xu, K. Fu et al., "Characterization and genomic analysis of quinolone-resistant Delftia sp. 670 isolated from a patient who died from severe pneumonia," Current Microbiology, vol. 71, no. 1, pp. 54-61, 2015.

[22] K. Blin, M. H. Medema, D. Kazempour et al., "antiSMASH 2.0a versatile platform for genome mining of secondary metabolite producers," Nucleic Acids Research, vol. 41, pp. W204-W212, 2013.

[23] J. Sambrook and D. W. Russell, Molecular Cloning: A Laboratory Manual, Cold Spring Harbor Laboratory Press, New York, NY, USA, 3rd edition, 2001.

[24] A. Machuca and A. M. F. Milagres, "Use of CAS-agar plate modified to study the effect of different variables on the siderophore production by Aspergillus," Letters in Applied Microbiology, vol. 36, no. 3, pp. 177-181, 2003.

[25] M. Manninen and T. Mattila-Sandholm, "Methods for the detection of Pseudomonas siderophores," Journal of Microbiological Methods, vol. 19, no. 3, pp. 223-234, 1994.

[26] B. Schwyn and J. B. Neilands, "Universal chemical assay for the detection and determination of siderophores," Analytical Biochemistry, vol. 160, no. 1, pp. 47-56, 1987.

[27] A. Baakza, A. K. Vala, B. P. Dave, and H. C. Dube, "A comparative study of siderophore production by fungi from marine and terrestrial habitats," Journal of Experimental Marine Biology and Ecology, vol. 311, no. 1, pp. 1-9, 2004.

[28] J. Felsenstein, "Evolutionary trees from DNA sequences: a maximum likelihood approach," Journal of Molecular Evolution, vol. 17, no. 6, pp. 368-376, 1981.

[29] A. W. Eastman, D. E. Heinrichs, and Z.-C. Yuan, "Comparative and genetic analysis of the four sequenced Paenibacillus polymyxa genomes reveals a diverse metabolism and conservation of genes relevant to plant-growth promotion and competitiveness," BMC genomics, vol. 15, article 851, 2014.

[30] D. Lu, J. Liu, and Z. Mao, "Engineering of Corynebacterium glutamicum to enhance L-ornithine production by gene knockout and comparative proteomic analysis," Chinese Journal of Chemical Engineering, vol. 20, no. 4, pp. 731-739, 2012.

[31] F. Xia, L. Zhang, S. Liang et al., "Study on root colonization of flowering Chinese cabbage by LuxAB gene-marked Pseudomonas flurosecens," Biotechnology, vol. 20, no. 1, pp. 25-27, 2010. 
[32] M. A. Wyatt, C. W. Johnston, and N. A. Magarvey, "Gold nanoparticle formation via microbial metallophore chemistries," Journal of Nanoparticle Research, vol. 16, no. 3, pp. 1-7, 2014.

[33] C. W. Johnston, M. A. Wyatt, X. Li et al., "Gold biomineralization by a metallophore from a gold-associated microbe," Nature Chemical Biology, vol. 9, no. 4, pp. 241-243, 2013. 

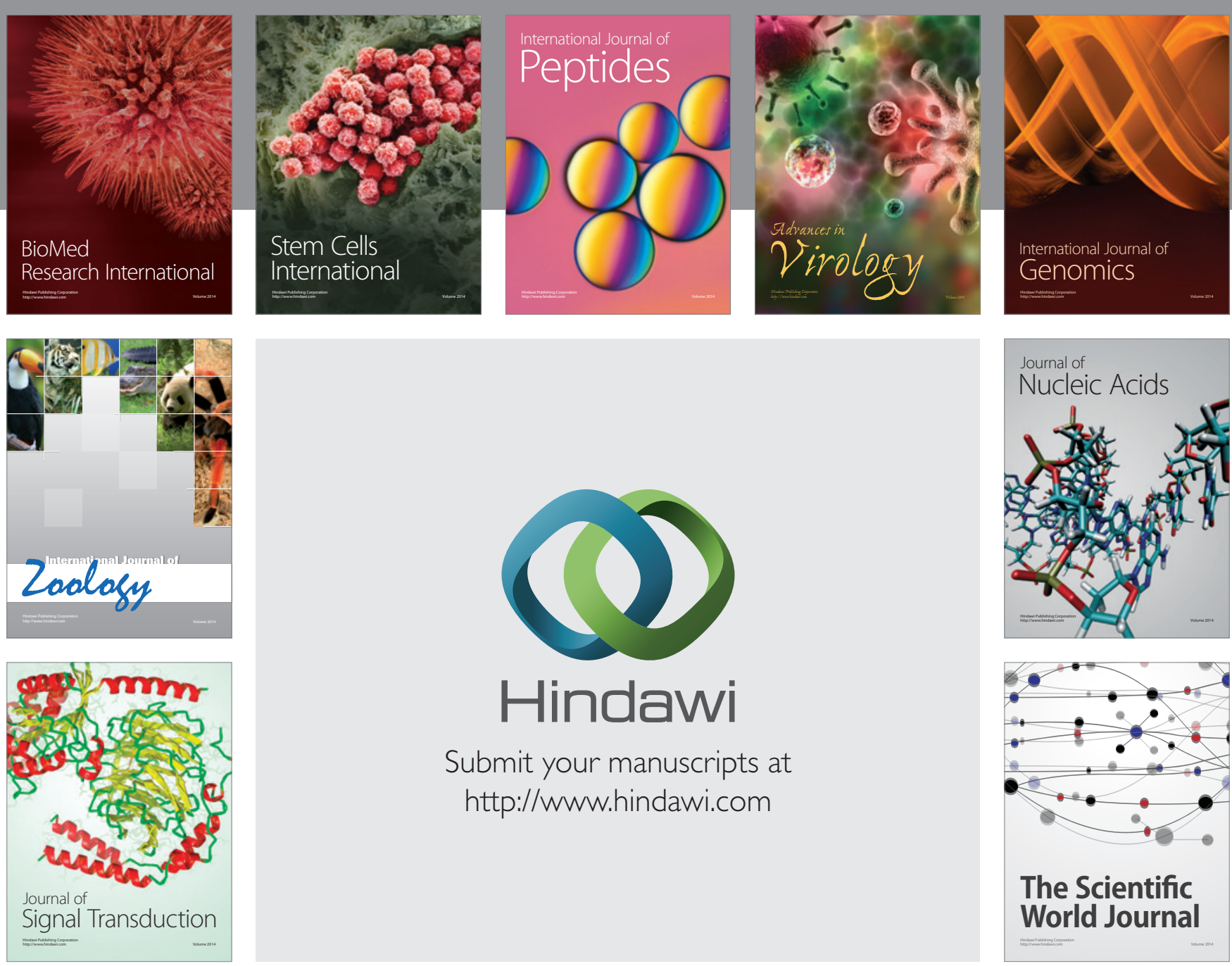

Submit your manuscripts at

http://www.hindawi.com
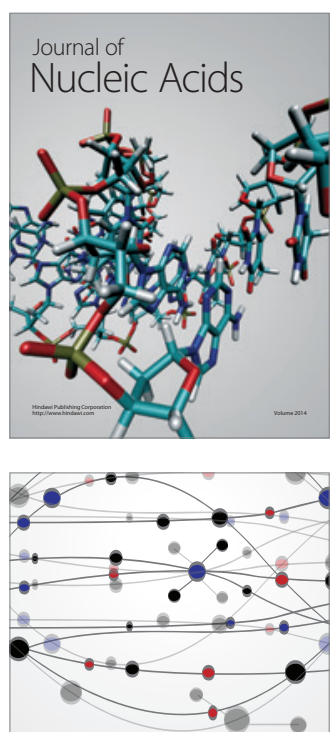

The Scientific World Journal
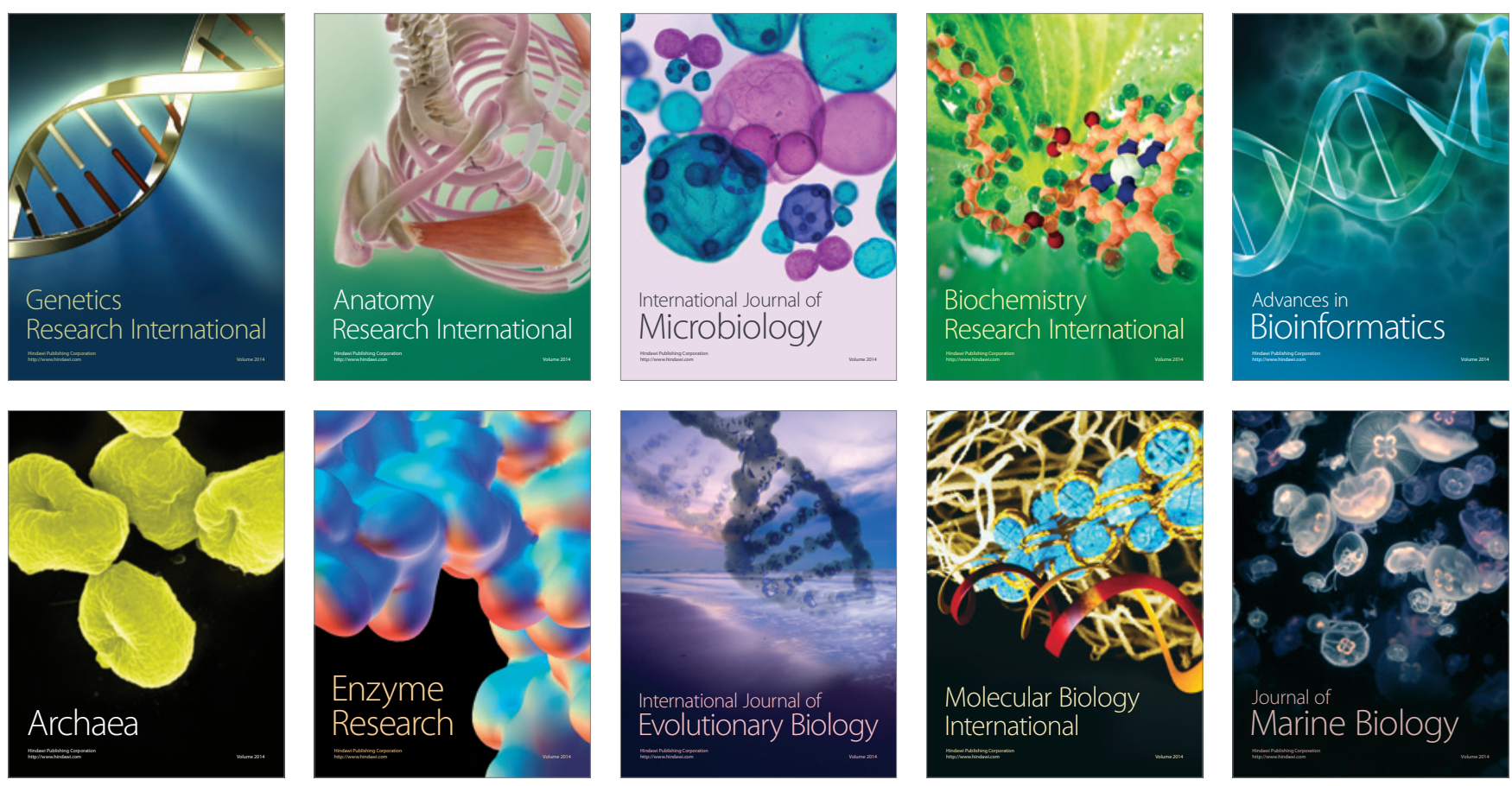